\title{
Baseline characteristics of patients enrolled in the Exenatide Study of Cardiovascular Event Lowering (EXSCEL)
}

Robert J. Mentz, MD, ${ }^{a}$ M. Angelyn Bethel, MD, ${ }^{b}$ Stephanie Gustavson, $\mathbf{P h D},{ }^{c}$ Vivian P. Thompson, MPH, ${ }^{a}$

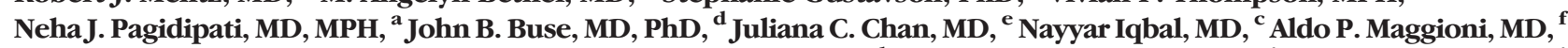
Steve P. Marso, MD, ${ }^{\mathrm{g}}$ Peter Ohman, MD, PhD, ${ }^{\mathbf{c}}$ Neil Poulter, FMedSci, ${ }^{\mathbf{h}}$ Ambady Ramachandran, $\mathrm{MD},{ }^{\mathbf{i}}$ Bernard Zinman, $\mathrm{MD},{ }^{\mathbf{j}}$

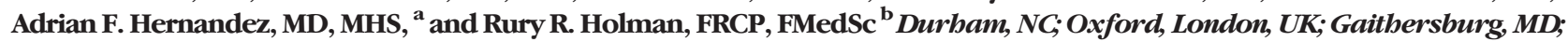
Chapel Hill, NG; Hong Kong, SAR, China; Florence, Itab; Dallas, TX; Chennai, India; and Ontario, Canada

Background EXSCEL is a randomized, double-blind, placebo-controlled trial examining the effect of exenatide onceweekly (EQW) versus placebo on time to the primary composite outcome (cardiovascular death, nonfatal myocardial infarction or nonfatal stroke) in patients with type 2 diabetes mellitus (DM) and a wide range of cardiovascular (CV) risk.

Methods Patients were enrolled at 688 sites in 35 countries. We describe their baseline characteristics according to prior $\mathrm{CV}$ event status and compare patients with those enrolled in prior glucagon-like peptide-1 receptor agonist (GLP-1 RA) outcomes trials.

Results Of a total of 14,752 participants randomized between June 2010 and September 2015, 6,788 (46.0\%) patients were enrolled in Europe; 3,708 (25.1\%), North America; 2,727 (18.5\%), Latin America; and 1,529 (10.4\%), Asia Pacific. Overall, $73 \%$ had at least one prior CV event $70 \%$ coronary artery disease, $24 \%$ peripheral arterial disease, $22 \%$ cerebrovascular disease). The median (IQR) age was 63 years $(56,69), 38 \%$ were female, median baseline $\mathrm{HbAlc}$ was $8.0 \%$ $(7.3,8.9)$ and $16 \%$ had a prior history of heart failure. Those without a prior CV event were younger with a shorter duration of diabetes and better renal function than those with at least one prior CV event. Compared with prior GLP-1RA trials, EXSCEL has a larger percentage of patients without a prior CV event and a notable percentage who were taking a dipeptidyl peptidase-4 inhibitor at baseline (15\%).

Conclusions EXSCEL is one of the largest global GLP-1RA trials, evaluating the safety and efficacy of EQW with a broad patient population that may extend generalizability compared to prior GLP-1 RA trials (ClinicalTrials.gov number, NCT01 144338). (Am Heart J 2017;187:1-9.)

Incretin-based medications including glucagon-like peptide-1 receptor agonists (GLP-1RAs) are routinely used for the treatment of type 2 diabetes mellitus

From the ${ }^{a}$ Duke Clinical Research Institute, Durham, NC, ${ }^{b}$ Diabetes Trials Unit, University of Oxford, Oxford, UK, 'AstraZeneca Research and Development, Gaithersburg, MD, 'University of North Carolina at Chapel Hill, Chapel Hill, NC, ${ }^{e}$ The Chinese University of Hong Kong, Hong Kong, SAR, China, ${ }^{f}$ ANMCO Research Centre, Florence, Italy, 'IUniversity of Texas Southwestern Medical Center, Dallas, TX, hInternational Centre for Circulatory Health, NHLI, Imperial College London, London, UK, IIndia Diabetes Research Foundation and Dr. A. Ramachandran's Diabetes Hospitals, Chennai, India, and 'Lunenfeld Tanenbaum Research Institute, Mount Sinai Hospital and University of Toronto, Toronto, Ontario, Canada. RCT\# NCTO 1144338

Frederick A Masoudi, MD, MSPH served as guest editor for this article.

Funding: The study is sponsored and funded by Amylin Pharmaceuticals Inc. (San Diego, CA), a wholly owned subsidiary of AstraZeneca (Gaithersburg, MD). Previous sponsors have included Eli Lilly and Company (Indianapolis, IN), and Bristol-Myers Squibb (Princeton, NJ). Submitted December 15, 2016; accepted February 8, 2017.

Reprint requests: Robert J. Mentz, MD, Duke Clinical Research Institute, PO Box 17969, Durham, NC 27715.

E-mail: robert.mentz@duke.edu

http://dx.doi.org/10.1016/i.ahj.2017.02.005
(T2DM). ${ }^{1}$ These medications effectively regulate glucose metabolism and have favorable effects on the myocardium and vascular system. ${ }^{2}$ GLP-1RAs may also have additional cardioprotective effects such as weight loss. ${ }^{3}$ Evidence from randomized clinical trials supports the cardiovascular safety of GLP-1RAs ${ }^{4}$ and recent clinical trial data with GLP-1RAs have shown an improvement in major adverse cardiac events (MACE) including cardiovascular death, non-fatal myocardial infarction (MI) and non-fatal stroke. ${ }^{5,6}$ However, a prior GLP-1RA trial was neutral with respect to MACE ${ }^{4}$ suggesting the lack of a class effect and highlighting the need for empiric evaluation of different agents in this class.

The EXSCEL trial is an academically led, placebo-controlled randomized trial of the GLP-1RA exenatide-once-weekly (EQW) in patients with $\mathrm{T}_{2} \mathrm{DM}^{7}$ that has recently completed enrollment. This is a pragmatic trial ${ }^{8}$ designed to assess the intervention in a real-world setting with broad entry criteria and streamlined trial conduct (eg, study visits every 6 months, laboratory evaluations as part of routine care) to enhance generalizability. The primary objective of EXSCEL is 
to evaluate the effect of EQW, used in addition to the current usual care for glycemic control and other $\mathrm{CV}$ risk factors, on major macrovascular events when administered to patients with T2DM. The study includes both a primary safety hypothesis, that EQW is non-inferior to usual care for MACE, and a primary efficacy hypothesis, that EQW is superior to usual care for MACE.

EXSCEL is unique in that it enrolled patients with a broad range of cardiovascular risk (including those without a prior cardiovascular event) and was not enriched for older people or other specific cardiovascular risk factors, allowed dual usage of incretin therapies [GLP-1 agonist investigational product and dipeptidyl peptidase-4 (DPP-4) inhibitor use] and did not include a run-in period often included to select more compliant patients than in routine practice. Additionally, there are both primary safety and efficacy hypotheses in EXSCEL in contrast to many other large T2DM cardiovascular outcomes trials with only a primary hypothesis of non-inferiority. ${ }^{4,5}$ In the present manuscript, we describe the baseline characteristics of EXSCEL participants according to their baseline prior cardiovascular event status and enrolling region and compare patients with those enrolled in prior large GLP-1RA trials.

\section{Methods}

The EXSCEL trial is an ongoing, multinational, doubleblind, placebo-controlled, randomized trial of the onceweekly GLP-1RA exenatide (EQW) at a dose of $2 \mathrm{mg}$ in addition to usual care for T2DM. The study background and design has been previously published. ${ }^{7}$ In brief, the trial enrolled participants with a broad range of cardiovascular risk, from 688 sites in 35 countries from North America, Latin America, Europe, and Asia Pacific. Randomization targeted approximately $70 \%$ with a history of a cardiovascular event and 30\% without known cardiovascular disease as detailed in the design manuscript. ${ }^{7}$ In brief, a prior cardiovascular event was defined as at least one of the following: a major clinical manifestation of coronary artery disease (CAD), ischemic cerebrovascular disease or atherosclerotic peripheral arterial disease (PAD). The trial has completed recruitment and is currently in the follow-up phase until the target event number of 1360 subjects with a confirmed primary composite outcome, defined as cardiovascular death, nonfatal myocardial infarction, or nonfatal stroke, has accrued.

In the present manuscript, baseline characteristics are presented in a blinded manner for the entire trial population according to prior cardiovascular event status at enrollment and by region. Regions were defined a priori (see Supplemental Table I for countries within each region) and broadly conform to regions defined in other studies in this field to facilitate comparison. We also compare EXSCEL patients with those enrolled in prior large GLP-1RA trials. ${ }^{4-6}$
No extramural funding was used to support this work. The authors are solely responsible for the design and conduct of this study, all study analyses, the drafting and editing of the manuscript, and its final contents.

\section{Results}

Overall, 14,752 participants were randomized between June 2010 and September 2015. The baseline characteristics of the full population are presented in Table I. The majority of patients enrolled in EXSCEL were white (75.8\%) with inclusion of 9.8\% Asian and 6.0\% Black. With regard to ethnicity, 3028 patients (20.5\%) reported being Hispanic or Latino. The median (IQR) age was 63 years $(56,69), 38 \%$ were female, and $16 \%$ had a prior history of heart failure (HF). The median (IQR) HbA1c, diabetes duration, and body mass index (BMI) were $8.0 \%$ $(7.3,8.9), 12(7,18)$ years, and $32(28,36) \mathrm{kg} / \mathrm{m}^{2}$, respectively. The most common anti-diabetic medication was metformin (76.5\%) followed by insulin (46.2\%) and sulfonylureas (36.6\%), and DPP-4 inhibitors (14.9\%). The baseline use of thiazolidinediones and SGLT-2 Inhibitors was low at $3.9 \%$ and $0.9 \%$, respectively.

In terms of World region, 6,788 (46.0\%) patients were enrolled in Europe; 3,708 (25.1\%), North America; 2,727 (18.5\%), Latin America; and 1529 (10.4\%), Asia Pacific. Figure 1 shows the enrollment by regions in EXSCEL. Supplemental Table I presents the number of sites and patients enrolled in each country. Table I presents baseline characteristics by World region. Patients from North America tended to be older with a longer duration of DM, a higher BMI and more CAD compared to other regions. Nearly half of the patients from Latin America were women and PAD was more prevalent in Latin America than other regions, yet statin and anti-platelet use was the lowest. Patients from Asia Pacific had the lowest BMI and had less usage of angiotensin converting enzyme (ACE)-inhibitors and angiotensin receptor blockers (ARBs) compared to other regions. In Europe, patients had the highest baseline blood pressure and nearly a quarter of patients had a prior history of HF.

With regard to baseline cardiovascular disease, $73 \%$ had at least one prior cardiovascular event (70\% CAD, 24\% PAD, 22\% cerebrovascular disease). Table II presents characteristics by baseline cardiovascular event status. The patients with a prior cardiovascular event tended to be older men with a longer duration of T2DM and worse renal function, but lower BMI and low-density lipoprotein (LDL) cholesterol, and similar blood pressure and HbA1c compared to those without known prior cardiovascular events at baseline. Compared to patients without a prior cardiovascular event, patients with a prior cardiovascular event had similar usage of DPP-4 inhibitors, less metformin and sulfonylurea use, and more insulin therapy. Evidence-based medications to address cardiovascular risk (eg, anti-hypertensive therapy, aspirin, 
Table I. Baseline characteristics by enrolling region

\begin{tabular}{|c|c|c|c|c|c|}
\hline & $\begin{array}{l}\text { All Regions } \\
N=14,752\end{array}$ & $\begin{array}{c}\text { North America } \\
N=3708\end{array}$ & $\begin{array}{c}\text { Latin America } \\
N=2727\end{array}$ & $\begin{array}{l}\text { Asia Pacific } \\
N=1529\end{array}$ & $\begin{array}{c}\text { Europe } \\
N=6788\end{array}$ \\
\hline Age $(y)$ & $62.7(56.4,68.8)$ & $64.1(57.4,70.3)$ & $61.5(55.0,68.0)$ & $61.4(55.1,67.4)$ & $62.7(56.8,68.6)$ \\
\hline $\operatorname{Min} / \max$ & $21.1,92.6$ & $23.6,89.9$ & $24.4,92.6$ & $25.7,87.9$ & $21.1,88.7$ \\
\hline$<40 y$ & $205(1.4 \%)$ & $56(1.5 \%)$ & $55(2.0 \%)$ & $30(2.0 \%)$ & $64(0.9 \%)$ \\
\hline 40 to $<65 y$ & $8606(58.3 \%)$ & $1912(51.6 \%)$ & $1689(61.9 \%)$ & $954(62.4 \%)$ & $4051(59.7 \%)$ \\
\hline 65 to $75 y$ & $4690(31.8 \%)$ & $1294(34.9 \%)$ & $777(28.5 \%)$ & $455(29.8 \%)$ & $2164(31.9 \%)$ \\
\hline$>75 y$ & $1251(8.5 \%)$ & 446 (12.0\%) & $206(7.6 \%)$ & $90(5.9 \%)$ & 509 (7.5\%) \\
\hline Women & 5604 (38.0\%) & $1142(30.8 \%)$ & $1341(49.2 \%)$ & 574 (37.5\%) & 2547 (37.5\%) \\
\hline \multicolumn{6}{|l|}{ Race } \\
\hline White & $11,175(75.8 \%)$ & $2800(75.6 \%)$ & 1494 (54.8\%) & $300(19.6 \%)$ & $6581(97.0 \%)$ \\
\hline Asian & $1452(9.8 \%)$ & $120(3.2 \%)$ & $7(0.3 \%)$ & $1212(79.3 \%)$ & $113(1.7 \%)$ \\
\hline Hispanic & $1134(7.7 \%)$ & 329 (8.9\%) & $799(29.3 \%)$ & $0(0.0 \%)$ & $6(0.1 \%)$ \\
\hline Black & $878(6.0 \%)$ & $430(11.6 \%)$ & $374(13.8 \%)$ & $1(0.1 \%)$ & $73(1.1 \%)$ \\
\hline Indian or Alaska Native & $73(0.5 \%)$ & $12(0.3 \%)$ & $51(1.9 \%)$ & $0(0.0 \%)$ & $10(0.1 \%)$ \\
\hline Pacific Islander or Hawaiian & $35(0.2 \%)$ & $14(0.4 \%)$ & $1(0.0 \%)$ & $16(1.0 \%)$ & $4(0.1 \%)$ \\
\hline \multicolumn{6}{|l|}{ Ethnicity } \\
\hline Hispanic or Latino & $3026(20.5 \%)$ & $366(9.9 \%)$ & $2564(94.0 \%)$ & $7(0.5 \%)$ & $89(1.3 \%)$ \\
\hline Non-Hispanic or Latino & $11,724(79.5 \%)$ & $3340(90.1 \%)$ & $163(6.0 \%)$ & $1522(99.5 \%)$ & $6699(98.7 \%)$ \\
\hline Diabetes duration $(y)$ & $12(7,18)$ & $13(7,19)$ & $11(6,18)$ & $11(7,18)$ & $11(7,17)$ \\
\hline$<5 y$ & $2018(13.7 \%)$ & $489(13.2 \%)$ & $454(16.7 \%)$ & $207(13.6 \%)$ & $868(12.8 \%)$ \\
\hline$\geq 5$ to $<15 y$ & $7264(49.4 \%)$ & $1693(45.9 \%)$ & $1248(45.8 \%)$ & $761(50.0 \%)$ & $3562(52.7 \%)$ \\
\hline$\geq 15 y$ & $5420(36.9 \%)$ & $1510(40.9 \%)$ & $1022(37.5 \%)$ & $554(36.4 \%)$ & $2334(34.5 \%)$ \\
\hline \multicolumn{6}{|l|}{ History of cardiovascular disease } \\
\hline Coronary artery disease & $7501(69.6 \%)$ & 2307 (86.7\%) & $1083(52.1 \%)$ & $811(70.8 \%)$ & $3300(67.4 \%)$ \\
\hline Cerebrovascular disease & 2419 (22.4\%) & $538(20.2 \%)$ & 267 (12.8\%) & $302(26.4 \%)$ & $1312(26.8 \%)$ \\
\hline Peripheral artery disease & $2540(23.6 \%)$ & $368(13.8 \%)$ & $918(44.1 \%)$ & $151(13.2 \%)$ & $1103(22.5 \%)$ \\
\hline History of retinopathy & $2511(17.0 \%)$ & $465(12.5 \%)$ & $347(12.7 \%)$ & $242(15.8 \%)$ & $1457(21.5 \%)$ \\
\hline History of heart failure & 2385 (16.2\%) & 403 (10.9\%) & $241(8.8 \%)$ & $77(5.0 \%)$ & $1664(24.5 \%)$ \\
\hline \multicolumn{6}{|l|}{ NYHA class } \\
\hline I & $736(30.9 \%)$ & $143(35.6 \%)$ & $106(44.0 \%)$ & $44(57.1 \%)$ & $443(26.7 \%)$ \\
\hline ॥ & $1333(55.9 \%)$ & 204 (50.7\%) & 114 (47.3\%) & $28(36.4 \%)$ & 987 (59.4\%) \\
\hline III & 301 (12.6\%) & $51(12.4 \%)$ & $19(7.9 \%)$ & $5(6.5 \%)$ & $226(13.6 \%)$ \\
\hline IV & $13(0.5 \%)$ & $5(1.2 \%)$ & $2(0.8 \%)$ & $0(0.0 \%)$ & $6(0.4 \%)$ \\
\hline \multicolumn{6}{|c|}{ Most recent assessment of LV function (ejection fraction) } \\
\hline Normal (>55\%) & $2924(59.8 \%)$ & 911 (60.2\%) & $331(62.5 \%)$ & $273(66.1 \%)$ & 1409 (57.9\%) \\
\hline Mild dysfunction (40-55\%) & $1497(30.7 \%)$ & $432(28.5 \%)$ & $135(25.5 \%)$ & $95(23.0 \%)$ & $835(34.3 \%)$ \\
\hline Moderate dysfunction (25-39\%) & $388(7.9 \%)$ & $134(8.9 \%)$ & $49(9.2 \%)$ & $36(8.7 \%)$ & $169(7.0 \%)$ \\
\hline Severe dysfunction (<25\%) & $81(1.7 \%)$ & $37(2.4 \%)$ & $15(2.8 \%)$ & $9(2.2 \%)$ & $20(0.8 \%)$ \\
\hline Systolic blood pressure (mmHg) & $135(124,145)$ & $130(120,140)$ & $135(122,149)$ & $131(121,144)$ & $138(129,147)$ \\
\hline Diastolic blood pressure $(\mathrm{mmHg})$ & $80(70-85)$ & $75(68-81)$ & $80(71-86)$ & $79(70-84)$ & $80(73-86)$ \\
\hline Heart rate (bpm) & $72(66,80)$ & $72(64,79)$ & $72(65,80)$ & $74(68,81)$ & $72(67,80)$ \\
\hline Body mass index $\left(\mathrm{kg} / \mathrm{m}^{2}\right)$ & $31.8(28.2,36.2)$ & $33.6(29.7,38.3)$ & $29.8(26.8,33.3)$ & $27.3(24.5,31.2)$ & $32.7(29.4,36.9)$ \\
\hline$<30 \mathrm{~kg} / \mathrm{m}^{2}$ & $5355(36.7 \%)$ & $976(26.8 \%)$ & $1388(51.1 \%)$ & $1036(69.0 \%)$ & $1955(29.0 \%)$ \\
\hline$\geq 30 \mathrm{~kg} / \mathrm{m}^{2}$ & 9247 (63.3\%) & $2668(73.2 \%)$ & $1328(48.9 \%)$ & $465(31.0 \%)$ & 4786 (71.0\%) \\
\hline $\mathrm{HbAlc}(\%)$ & $8.0(7.3,8.9)$ & $7.9(7.3,8.7)$ & $8.1(7.4,9.1)$ & $8.0(7.4,8.9)$ & $8.0(7.3,8.9)$ \\
\hline $\operatorname{Min} / \max$ & $5.6,12.7$ & $5.6,12.7$ & $5.9,10.9$ & $6.1,12.6$ & $6.0,11.7$ \\
\hline Mean (SD) in patients $<8 \%$ & $7.3(0.4)$ & $7.3(0.4)$ & $7.3(0.4)$ & $7.3(0.4)$ & $7.3(0.4)$ \\
\hline Mean (SD) in patients $\geq 8 \%$ & $8.9(0.6)$ & $8.9(0.6)$ & $9.0(0.6)$ & $8.9(0.7)$ & $8.9(0.6)$ \\
\hline $\mathrm{LDL}(\mathrm{mg} / \mathrm{dL})$ & $88(66,116)$ & $76(60,98)$ & $100(74,128)$ & $84(65,110)$ & $93(71,124)$ \\
\hline eGFR via MDRD (mL/min per $\left.1.73 \mathrm{~m}^{2}\right)$ & $79(64,96)$ & $78(62,95)$ & $78(64,93)$ & $81(64,99)$ & $81(66,96)$ \\
\hline$>90$ & $5036(34.2 \%)$ & $1214(32.7 \%)$ & $854(31.3 \%)$ & $567(37.1 \%)$ & $2401(35.4 \%)$ \\
\hline $60-89$ & $6953(47.2 \%)$ & $1684(45.4 \%)$ & $1342(49.2 \%)$ & $687(45.0 \%)$ & $3240(47.8 \%)$ \\
\hline $30-59$ & 2743 (18.6\%) & $807(21.8 \%)$ & $527(19.3 \%)$ & $270(17.7 \%)$ & $1139(16.8 \%)$ \\
\hline \multicolumn{6}{|l|}{ Anti-DM therapies } \\
\hline Metformin & 11,289 (76.5\%) & $2617(70.6 \%)$ & 2250 (82.5\%) & $1164(76.1 \%)$ & $5258(77.5 \%)$ \\
\hline Sulfonylurea & $5402(36.6 \%)$ & $1416(38.2 \%)$ & $1144(42.0 \%)$ & $659(43.1 \%)$ & 2183 (32.2\%) \\
\hline Thiazolidinedione & 579 (3.9\%) & $342(9.2 \%)$ & $21(0.8 \%)$ & $74(4.8 \%)$ & $142(2.1 \%)$ \\
\hline DPP-4 inhibitor & 2202 (14.9\%) & 641 (17.3\%) & $211(7.7 \%)$ & $240(15.7 \%)$ & 1110 (16.4\%) \\
\hline SGLT-2 inhibitor & 77 (0.9\%) & 15 (0.9\%) & $10(0.5 \%)$ & $3(0.3 \%)$ & 49 (1.3\%) \\
\hline
\end{tabular}


fable I (eontinued)

\begin{tabular}{lccccc}
\hline & All Regions & North America & Latin America & Asia Pacific & Europe \\
$N=14,752$ & $N=3708$ & $N=2727$ & $N=1529$ & $N=6788$ \\
\hline Insulin & $6819(46.2 \%)$ & $1591(42.9 \%)$ & $1215(44.6 \%)$ & $698(45.7 \%)$ & $3315(48.8 \%)$ \\
$\quad$ And 0-2 oral AHAs & $6738(45.7 \%)$ & $1575(42.5 \%)$ & $1202(44.1 \%)$ & $689(45.1 \%)$ & $3272(48.2 \%)$ \\
$\quad$ And $>$ oral AHAs & $81(0.5 \%)$ & $16(0.4 \%)$ & $13(0.5 \%)$ & $9(0.6 \%)$ & $43(0.6 \%)$ \\
Monotherapy (including oral agents and insulin) & $2687(32.0 \%)$ & $572(33.7 \%)$ & $640(32.0 \%)$ & $236(25.0 \%)$ & $1239(33.0 \%)$ \\
2 oral therapies & $1914(46.8 \%)$ & $338(46.3 \%)$ & $569(53.7 \%)$ & $195(47.2 \%)$ & $812(43.1 \%)$ \\
No glucose lowering therapy & $125(1.5 \%)$ & $35(2.1 \%)$ & $24(1.2 \%)$ & $18(1.9 \%)$ & $48(1.3 \%)$ \\
Cardiovascular medications & & & & \\
Aspirin & $9372(63.5 \%)$ & $2685(72.5 \%)$ & $1594(58.5 \%)$ & $920(60.2 \%)$ & $4173(61.5 \%)$ \\
Thienopyridines & $2521(17.1 \%)$ & $730(19.7 \%)$ & $333(12.2 \%)$ & $322(21.1 \%)$ & $1136(16.7 \%)$ \\
Any anti-platelets & $10,310(69.9 \%)$ & $2871(77.5 \%)$ & $1659(60.8 \%)$ & $1089(71.2 \%)$ & $4691(69.1 \%)$ \\
ACEl or ARB & $11,386(77.2 \%)$ & $2881(77.7 \%)$ & $1938(71.1 \%)$ & $1029(67.3 \%)$ & $5538(81.6 \%)$ \\
B-Blockers & $8208(55.6 \%)$ & $2166(58.4 \%)$ & $1085(39.8 \%)$ & $714(46.7 \%)$ & $4243(62.5 \%)$ \\
Calcium-channel blocker & $4701(31.9 \%)$ & $1024(27.6 \%)$ & $609(22.3 \%)$ & $592(38.7 \%)$ & $2476(36.5 \%)$ \\
Any anti-hypertensive & $13,318(90.3 \%)$ & $3424(92.4 \%)$ & $2220(81.4 \%)$ & $1320(86.3 \%)$ & $6354(93.6 \%)$ \\
Statin & $10,836(73.5 \%)$ & $3094(83.5 \%)$ & $1617(59.3 \%)$ & $1143(74.8 \%)$ & $4982(73.4 \%)$ \\
Any lipid-lowering Rx & $11,364(77.0 \%)$ & $3210(86.6 \%)$ & $1746(64.0 \%)$ & $1197(78.3 \%)$ & $5211(76.8 \%)$ \\
\hline
\end{tabular}

$A H A$, Anti-hyperglycemic agents; $H b A l c$, hemoglobin Alc; $L D L$, low density lipoprotein; eGFR, estimated glomerular filtration rate; MDRD, modification of diet in renal disease; DPP-IV, dipeptidyl peptidase; SGLT-2, sodium-glucose co-transporter 2; ACEI, angiotensin converting enzyme inhibitor; ARB, angiotensin receptor blocker.

\section{Figure 1}

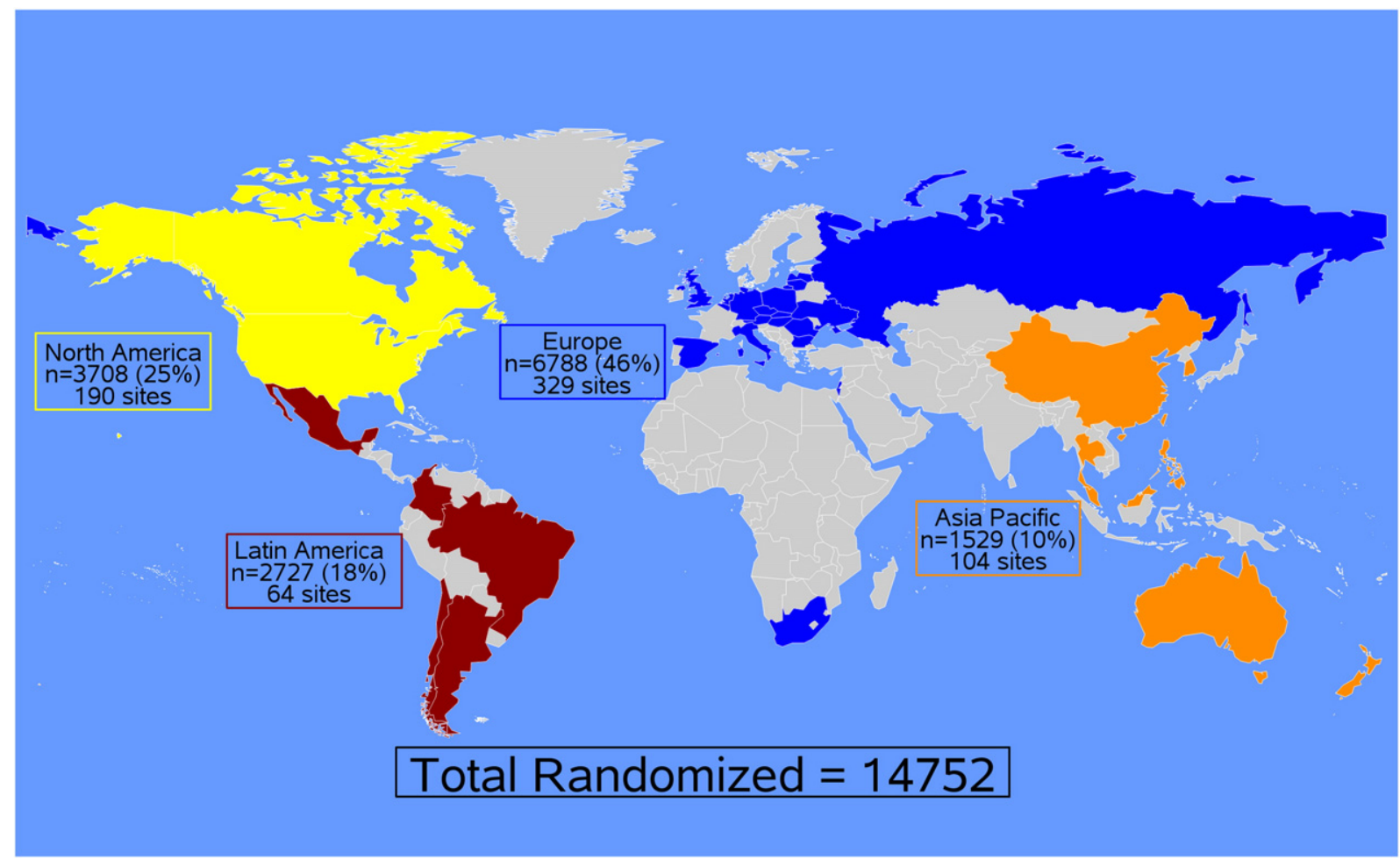

Enrollment of EXSCEL trial participants by World Region.

statin) were used more frequently in the secondary prevention population. Nonetheless, the primary prevention population demonstrated $81 \%$ use of an anti-hypertensive, $57 \%$ statin use and 38\% aspirin use at baseline.
Table III presents the EXSCEL design and baseline characteristics compared to prior cardiovascular outcomes studies with GLP-1RAs. Notable differences include the lack of an age criterion in EXSCEL and a primary efficacy hypothesis of superiority. 
Table II. Baseline characteristics by baseline CV status

No Prior Known CV Disease $N=3969$

Age (years)
Min/max
$<40$ y
40 to $<65$ y
65 to $75 y$
$>75 y$
Women
Race
White
Asian
Hispanic
Black
Indian or Alaska Native
Pacific Islander or Haw
Ethnicity
Hispanic or Latino
Non-Hispanic or Latino
DM duration (y)
$<5$ y
$\geq 5$ to $<15 y$
$\geq 15 y$
History of retinopathy
History of heart failure
NYHA class
I
II
III
IV

Most recent assessment of LV function (ejection fraction) Normal $(>55 \%)$

Mild dysfunction (40-55\%)

Moderate dysfunction (25-39\%)

Severe dysfunction $(<25 \%)$

Systolic BP (mmHg)

Diastolic BP (mmHg)

Heart rate (bpm)

BMI $\left(\mathrm{kg} / \mathrm{m}^{2}\right)$

$<30 \mathrm{~kg} / \mathrm{m}^{2}$

$\geq 30 \mathrm{~kg} / \mathrm{m}^{2}$

$\mathrm{HbAlc}(\%)$

LDL $(\mathrm{mg} / \mathrm{dL})$

eGFR via $M D R D\left(\mathrm{~mL} / \mathrm{min}\right.$ per $\left.1.73 \mathrm{~m}^{2}\right)$

$>90$

60-89

30-59

Anti-DM therapies

Metformin

Sulfonylurea

Thiazolidinedione

DPP-4 Inhibitor

SGLT-2 Inhibitor

Insulin

And 0-2 oral AHAs

And $>2$ oral AHAs

Monotherapy (including oral agents and insulin)

No glucose lowering therapy

Cardiovascular medications

Aspirin

Thienopyridines

Any anti-platelets

ACEI or ARB

$$
\begin{gathered}
59.4(52.5,65.7) \\
21.1,92.6 \\
150(3.8 \%) \\
2730(68.8 \%) \\
902(22.7 \%) \\
187(4.7 \%) \\
2023(51.0 \%) \\
\\
3040(76.6 \%) \\
327(8.2 \%) \\
331(8.3 \%) \\
251(6.3 \%) \\
10(0.3 \%) \\
9(0.2 \%)
\end{gathered}
$$

$755(19.0 \%)$

$3214(81.0 \%)$

$10(6,15)$

$703(17.7 \%)$

$2180(55.0 \%)$

$1078(27.2 \%)$

$467(11.8 \%)$

$321(8.1 \%)$

$119(37.1 \%)$

$172(53.6 \%)$

$30(9.3 \%)$

$0(0.0 \%)$

$477(77.2 \%)$

$118(19.1 \%)$

$18(2.9 \%)$

$5(0.8 \%)$

$134(124,144)$

$80(74-86)$

$75(68,81)$

$33.0(29.1,37.9)$

$1213(30.9 \%)$

$2715(69.1 \%)$

$8.0(7.4,8.9)$

$99(75,126)$

$85(71,103)$

$1718(43.3 \%)$

$1774(44.7 \%)$

$473(11.9 \%)$

3375 (85.1\%)

$1708(43.0 \%)$

$278(7.0 \%)$

$692(17.4 \%)$

24 (1.4\%)

1257 (31.7\%)

$1240(31.2 \%)$

$17(0.4 \%)$

$444(26.9 \%)$

37 (2.2\%)

1525 (38.4\%)

$64(1.6 \%)$

$1600(40.3 \%)$

$2765(69.7 \%)$
Prior CV Disease

$N=10,781$

$63.9(57.9,69.7)$

$25.7,90.2$

$54(0.5 \%)$

$5875(54.5 \%)$

$3788(35.1 \%)$

1064 (9.9\%)

3580 (33.2\%)

8133 (75.5\%)

1125 (10.4\%)

803 (7.5\%)

$627(5.8 \%)$

$63(0.6 \%)$

$26(0.2 \%)$

2271 (21.1\%)

8508 (78.9\%)

$12(7,19)$

$1315(12.2 \%)$

$5083(47.3 \%)$

$4341(40.4 \%)$

2044 (19.0\%)

2064 (19.1\%)

$617(29.9 \%)$

$1161(56.3 \%)$

271 (13.1\%)

$13(0.6 \%)$

2447 (57.3\%)

$1379(32.3 \%)$

$370(8.7 \%)$

$76(1.8 \%)$

$135(124,146)$

79 (70-84)

$72(64,79)$

$31.4(28.0,35.6)$

$4142(38.8 \%)$

$6531(61.2 \%)$

$8.0(7.3,8.9)$

$84(64,112)$

$77(62,93)$

3317 (30.8\%)

5179 (48.1\%)

$2269(21.1 \%)$

7914 (73.4\%)

3694 (34.3\%)

301 (2.8\%)

$1510(14.0 \%)$

$53(0.8 \%)$

$5562(51.6 \%)$

$5498(51.0 \%)$

$64(0.6 \%)$

$2243(33.2 \%)$

$88(1.3 \%)$

7847 (72.8\%)

$2457(22.8 \%)$

$8710(80.8 \%)$

$8621(80.0 \%)$ 


\begin{tabular}{lcc}
\hline & No Prior Known CV Disease & $\begin{array}{c}\text { Prior CV Disease } \\
\mathrm{N}=10,781\end{array}$ \\
\hline -Blockers & $1263(31.8 \%)$ & $6945(64.4 \%)$ \\
Calcium channel blocker & $1060(26.7 \%)$ & $3641(33.8 \%)$ \\
Any anti-hypertensive & $3197(80.5 \%)$ & $10,121(93.9 \%)$ \\
Statin & $2260(56.9 \%)$ & $8576(79.5 \%)$ \\
Any lipid-lowering Rx & $2473(62.3 \%)$ & $8891(82.5 \%)$ \\
\hline
\end{tabular}

\section{Discussion}

The EXSCEL trial enrolled more than 14,000 patients worldwide in order to assess the safety and efficacy of the GLP-1RA EQW. Nearly half of the patients were enrolled in Europe with significant contributions from North America (25\%), Latin America (19\%), and Asia Pacific (10\%). After completion of enrollment, there were notable differences between regions with respect to baseline characteristics and pharmacotherapy for both DM and cardiovascular disease. EXSCEL is one of the first large-scale outcome trials with a GLP-1RA to allow co-therapy with DPP- 4 inhibitors and approximately $15 \%$ of patients received these agents at baseline. EXSCEL was also unique in the inclusion of patients with a broad range of cardiovascular risk including approximately 30\% without known cardiovascular disease and nearly $50 \%$ on insulin at baseline.

EXSCEL has several distinct features related to the enrolled patient population compared to previous GLP-1RA outcomes trials (Table III). The ELIXA trial (Evaluation of LIXisenatide in Acute Coronary Syndrome) investigated the GLP-1RA lixisenatide compared with placebo in 6068 patients with an acute coronary syndrome (myocardial infarction in $83 \%$ and unstable angina in $17 \%$ ) within the previous 180 days 9 . Thus, only secondary prevention patients were included in the trial. Patients in ELIXA were younger (60 vs 63 years) with lower BMI (30 vs $32 \mathrm{~kg} / \mathrm{m}^{2}$ ) and shorter duration of diabetes ( 9 years vs 12 years) compared with patients in EXSCEL. ELIXA also incorporated a run-in period prior to randomization which selected for the more compliant patients, and thus may limit broader generalizability. ELIXA met its primary endpoint of non-inferiority for MACE- 4 but not superiority over a median follow-up of 2.1 years.

The LEADER trial investigated liraglutide compared with placebo in 9340 diabetics at high risk for cardiovascular events. ${ }^{10}$ Patients were required to be $\geq 50$ years at screening in contrast to EXSCEL which did not have an age criterion beyond adulthood (18 years). Overall, 81\% of LEADER patients were identified as having prior cardiovascular disease and $19 \%$ were without prior cardiovascular disease compared to $27 \%$ without a prior cardiovascular event in EXSCEL. However, the definition of prior cardiovascular disease in LEADER also included those with renal impairment or chronic HF in comparison to the requirement in EXSCEL for at least one of the following: CAD, PAD or ischemic cerebrovascular disease. Moreover, the LEADER trial enriched the primary prevention cohort through additional requirements (e.g., $\geq 60$ years and concomitant disease history of either documented nephropathy, underlying cardiac abnormalities [left ventricular hypertrophy or ventricular dysfunction] or evidence of PAD). Similar to ELIXA, LEADER also incorporated a run-in period prior to randomization. This design characteristic as well as the risk factor enrichment for the subgroup without prior cardiovascular disease may limit generalization to the broader population of patients with type 2 diabetes.

Patients in LEADER had a similar mean age (64 years) and BMI $\left(32.5 \mathrm{~kg} / \mathrm{m}^{2}\right)$ compared to the EXSCEL cohort but the baseline HbA1c was higher in LEADER (8.7\% vs 8.0\%). LEADER met its primary endpoint of non-inferiority for MACE-3 and also demonstrated superiority (HR $0.87,95 \%$ CI: $0.78-97, P=.01$ for superiority) over a median follow-up of 3.8 years. Therefore, LEADER was the first GLP-1 agonist outcome study to demonstrate superiority for cardiovascular outcomes.

The SUSTAIN-6 trial investigated the GLP-1RA semaglutide in 3297 patients with T2DM. ${ }^{6}$ Eligible patients were $\geq 50$ years old with clinical evidence of cardiovascular disease or $\geq 60$ years old with subclinical cardiovascular disease. The SUSTAIN-6 patient population had the oldest age of the GLP-1RA outcomes trials completed to date and had the longest duration of DM as well as the highest usage of insulin at baseline. With a 104-week treatment period, the trial met its primary endpoint of non-inferiority for MACE (HR 0.74; 95\% CI: 0.58-0.95, $P<.001$ ) as well as a statistical significant reduction in CV risk with 254 primary MACE events. There was a significant reduction in non-fatal stroke, a numerical reduction in non-fatal MI and a neutral relationship with CV death. Thus, SUSTAIN-6 included an age entry criterion and targeted patients with known $\mathrm{CV}$ or kidney disease or $\mathrm{CV}$ risk factors with a modest event count over an observation period of approximately 2 years.

Importantly, certain effects of the GLP-1RAs appear to represent class effects while there are also unique aspects of their efficacy and safety that may differ within this class 
Table III. Prior global cardiovascular outcomes trials for incretin-based therapies compared with EXSCEL

\begin{tabular}{|c|c|c|c|c|}
\hline $\begin{array}{l}\text { Design and baseline } \\
\text { characteristics }\end{array}$ & ELIXA & LEADER & SUSTAIN-6 & EXSCEL \\
\hline Treatment & Lixisenatide & Liraglutide & Semaglutide & Exenatide \\
\hline No. of patients & 6068 & 9340 & 3297 & 14,752 \\
\hline No. of primary events & 805 & 1302 & 254 & Targeting 1360 \\
\hline \multirow[t]{3}{*}{ Key entry criteria } & Alc $5.5-11 \%$ & $\mathrm{Alc} \geq 7 \%$ & $\mathrm{Alc} \geq 7 \%$ & Alc $6.5-10.0 \%$ \\
\hline & ACS within 180 days & Established CVD & Established & Established CVD \\
\hline & $\geq 30$ yо & $\begin{array}{l}\text { and/or multiple } \\
\text { risk factors }\end{array}$ & $\begin{array}{l}\text { CVD and/or } \\
\text { multiple risk } \\
\text { factors }\end{array}$ & $\begin{array}{l}\text { as well as primary } \\
\text { prevention } \\
\text { ( } 27 \% \text { enrolled) }\end{array}$ \\
\hline Age criterion & $\geq 30 y$ & $\geq 50$ years with & $\geq 50$ years with & No specific age criterion \\
\hline & & $\begin{array}{l}\text { CV disease, } \\
\text { renal dysfunction } \\
\text { or HF or } \geq 60 \text { years } \\
\text { with at least one } \\
\text { additional high-risk } \\
\text { feature }\end{array}$ & $\begin{array}{l}\text { CVD or } \geq 60 \text { years } \\
\text { with subclinical CVD }\end{array}$ & \\
\hline $\begin{array}{l}\text { Duration of } \\
\text { follow up (median) }\end{array}$ & 2.1 y & 3.8 y & $2.1 \mathrm{y}$ & Pending \\
\hline Baseline Alc (\%) & $7.7 \pm 1.3$ (active arm) & $8.7 \pm 1.5$ & $8.7 \pm 1.5$ & $8.0(7.3,8.9)$ \\
\hline \multirow[t]{2}{*}{ Primary endpoint } & MACE-4 & MACE-3 & MACE-3 & MACE-3 \\
\hline & (non-inferiority) & (non-inferiority) & (non-inferiority) & $\begin{array}{l}\text { (superiority)/non-inferiority } \\
\text { for safety }\end{array}$ \\
\hline \multirow{3}{*}{$\begin{array}{l}\text { Results } \\
\text { [HR }(95 \% \mathrm{Cl})]\end{array}$} & HR 1.02 (0.89-1.17) & HR 0.87 (0.78-0.97) & HR 0.74 (0.58-0.95) & Pending \\
\hline & $P<.01$ for $\mathrm{NI}$ & $P<.001$ for $\mathrm{Nl}$ & $P<.001$ for $\mathrm{NI}$ & \\
\hline & $P=N S$ for superiority & $P=.01$ for superiority & & \\
\hline Age (y) & 60 & 64 & 65 & 63 \\
\hline Men & $70 \%$ & $64 \%$ & $61 \%$ & $62 \%$ \\
\hline DM duration (y) & 9.3 & 13 & 14 & 12 \\
\hline BMl $\left(\mathrm{kg} / \mathrm{m}^{2}\right)$ & 30 & 33 & 33 & 32 \\
\hline White & $75 \%$ & $78 \%$ & $83 \%$ & $76 \%$ \\
\hline Asian & $12 \%$ & $10 \%$ & $8 \%$ & $10 \%$ \\
\hline GFR $\left(\mathrm{mL} / \mathrm{min}\right.$ per $\left.1.73 \mathrm{~m}^{2}\right)$ & 75 & NR & NR & 79 \\
\hline Insulin & $39 \%$ & $42 \%$ & $58 \%$ & $46 \%$ \\
\hline Metformin & $66 \%$ & $76 \%$ & $73 \%$ & $77 \%$ \\
\hline SU & $33 \%$ & $51 \%$ & $43 \%$ & $37 \%$ \\
\hline TZD & $2 \%$ & $6 \%$ & $2 \%$ & $4 \%$ \\
\hline Aspirin & $97 \%$ antiplatelet & $63 \%$ & $64 \%$ & $64 \%$ \\
\hline Statin & $93 \%$ & $72 \%$ & $73 \%$ & $74 \%$ \\
\hline ACE-I/ARB & $85 \%$ & $51 \% / 32 \%$ & $50 \% / 34 \%$ & $77 \%$ \\
\hline
\end{tabular}

$D M$, Diabetes mellitus; $B M I$, body mass index; GFR, glomerular filtration rate; $S U$, sulfonylurea; TZD, thiazolidinedione; $A C E-A R B$, angiotensin converting enzyme inhibitor angiotensin receptor blocker; GLP-1, glucagon-like peptide-1; DPP-4, dipeptidyl peptidase-4. MACE-3, CV-related death, non-fatal MI, or non-fatal stroke.

$M A C E-4, C V$-related death, non-fatal MI, non-fatal stroke, or UA requiring hospitalization.

as reviewed previously. ${ }^{11}$ Overall, GLP-1RAs improve glycemic control while reducing body weight and systolic blood pressure. Moreover, due to their glucosedependent mechanism, the incidence of hypoglycemia is relatively low with these treatments compared to other anti-hyperglycemic therapies. In terms of duration of action, there are currently short-acting formulations that are administered once or twice daily (eg, exenatide, liraglutide and lixisenatide) compared with long-acting formulations that are injected weekly (eg, exenatide, albiglutide, dulaglutide). Long-acting agents may produce superior glucose lowering with less nausea than the shorter acting therapies. Comparative effectiveness data with regard to clinical outcomes and safety are needed within this medication class.
Differences in baseline characteristics were observed across enrolling regions in EXSCEL. One notable international variation was the baseline prevalence of $\mathrm{HF}$. The overall trial prevalence was $16 \%$ with regional values ranging from 5\% in Asia Pacific to $25 \%$ in Europe. Importantly, HF was identified by patient-reported medical history at baseline (ie, including past and present $\mathrm{HF}$ ) and did not require documentation of natriuretic peptide level or left ventricular ejection fraction. Prior studies in the HF literature have highlighted marked regional variation in HF diagnosis, severity and clinical outcomes. ${ }^{12,13}$ Information on baseline HF prevalence is particularly relevant given the uncertainty of the effects of incretin-based therapy in patients with HF. In the recent large-scale T2DM outcomes trial with the DPP-4 
inhibitor saxagliptin (SAVOR), there were observations of increased hospitalization for HF. ${ }^{14,15}$ Increased hospitalization for HF has not been observed consistently with other DPP-4 inhibitors indicating the lack of a class effect. Specific data assessing HF effects with GLP-1RAs have also varied from no effect with lixisenatide ${ }^{4}$ or semaglutide $^{6}$ to a nominal reduction in HF events with liraglutide. ${ }^{5}$ Reduced HF events were demonstrated with the sodiumglucose co-transporter 2 (SGLT2) inhibitor empagliflozin. ${ }^{16,17}$ Therefore, the HF-related findings from EXSCEL will provide much needed data regarding DM management in HF patients and/or those at increased risk for $\mathrm{HF}$.

\section{Limitations}

Baseline characteristics were presented by world region using country groupings similar to prior cardiovascular outcome studies; however, there may be significant patient and provider level variation even within the same region. Additionally, baseline characteristics were presented by prior cardiovascular disease status at randomization, the classification of which was determined by site investigators using available data regarding patients' medical history. However, there is the potential for misclassification of cardiovascular risk given that source documentation was not required for this determination. Moreover, the trial was pragmatic in nature and baseline labs were documented from routine care and not specifically drawn for the study. Nonetheless, these data provide important insights regarding the patient population of the multinational EXSCEL trial.

\section{Conclusions}

EXSCEL is one of the largest GLP-1RA trials, evaluating the effect of once weekly exenatide on cardiovascular safety and efficacy. Nearly half of the patients were enrolled in Europe with significant contributions from North America, Latin America, and Asia Pacific. Unique characteristics include a substantial percentage of patients with no prior cardiovascular event, and a notable percentage who were taking a DPP-4 inhibitor at baseline. The pragmatic design will support the generalizability of the data due to the lack of an upper age limit, no run-in period, the inclusion of primary and secondary cardiovascular prevention patients, as well as a wide range of background DM medications.

Supplementary data to this article can be found online at http://dx.doi.org/10.1016/j.ahj.2017.02.005.

\section{Disclosures}

RJM receives research support from the National Institutes of Health (U10HL110312 and R01AG045551-01A1), AstraZeneca, Bristol-Myers Squibb, and GlaxoSmithKline; and has served on an advisory board for Boehringer Ingelheim. M.A.B. has received research funding from Novartis and Bayer. SG, NI, and PO are employees of AstraZeneca. J.B.B. is an investigator and/or consultant without any direct financial benefit under contracts between his employer and the following companies: Amylin Pharmaceuticals, Inc., Andromeda, Astellas, Astra Zeneca, Bayhill Therapeutics, Inc., Boehringer Ingelheim GmbH \& Co KG, Bristol-Myers Squibb Company, Catabasis, Cebix, Inc., CureDM, Diartis Pharmaceuticals, Elcelyx Therapeutics, Inc., Eli Lilly and Company, Exsulin, Genentech, GI Dynamics, GlaxoSmithKline, Halozyme Therapeutics, F. Hoffmann-La Roche, Ltd., Intarcia Therapeutics, Johnson \& Johnson, Lexicon, LipoScience, Macrogenics, Medtronic, Merck, Metabolic Solutions Development Co, Metabolon, Inc., Metavention, Novan, Novo Nordisk A/S, Orexigen Therapeutics, Inc., Osiris Therapeutics, Inc., Pfizer, Inc., Quest Diagnostics, Rhythm Pharmaceuticals, Sanofi, Spherix, Inc., Takeda, ToleRx, Transpharma Medical Ltd., TransTech Pharma, Veritas, and Verva. He is a paid consultant to PhaseBio Pharmaceuticals Inc. and has received stock options for that effort. J.C.C. received research grant and/or honoraria for consultancy and/or giving lectures from AstraZeneca, Bayer, Boehringer Ingelheim, Eli Lilly, GlaxoSmithKline, Merck Sharp \& Dohme, Novo Nordisk, Pfizer, and/or Sanofi. All proceeds have been donated to the Chinese University of Hong Kong to support research and education. The Chinese University of Hong Kong has received research grants and sponsorships from these companies. A.P.M. reports personal honoraria for research activities from Novartis, Bayer, Pfizer, and Cardiorentis outside the submitted work. S.P.M. reports grants from The Medicines Company, Novo Nordisk, Abbott Vascular, Amylin Pharmaceuticals, Boston Scientific, Volcano Corporation, and Terumo Medical. He has received consulting fees from Novo Nordisk and St. Jude Medical. N.P. has received research grants from Servier and speaking honoraria from Servier, Novo Nordisk, and Amgen. A.R. received remuneration for Advisory board meetings from Merck, Sharp \& Dohme, and AstraZeneca; honoraria for lectures from Bayer, Novo Nordisk, Eli Lilly, Merck, Sharp \& Dohme, and Novartis; and research grant from Merck, Sharp \& Dohme. B.Z. reports grants support from Merck, Boehringer Ingelheim, and Novartis. He serves on medical advisory boards for Eli Lilly, Merck, Novo Nordisk, Janssen, Takeda, and Astra Zeneca. A.F.H. has received research grants from AstraZeneca, Bristol-Myers Squibb, Janssen, and Novartis and honoraria from Bristol-Myers Squibb and Janssen. R.R.H. has received research support from Bayer, BMS, and Merck; attended advisory boards with Amgen, Bayer, Elcelyx, Merck, Novartis, and Novo Nordisk; and given lectures supported by Bayer. The remaining authors report no conflicts of interest related to this manuscript.

\section{References}

1. Lovshin JA, Drucker DJ. Incretin-based therapies for type 2 diabetes mellitus. Nat Rev Endocrinol 2009;5(5):262-9. 
2. Ussher JR, Drucker DJ. Cardiovascular actions of incretin-based therapies. Circ Res 2014;114(11):1788-803.

3. Waldrop G, Zhong J, Peters M, et al. Incretin-Based Therapy for Diabetes: What a Cardiologist Needs to Know. J Am Coll Cardiol 2016;67(12):1488-96.

4. Pfeffer MA, Claggett B, Diaz R, et al. Lixisenatide in Patients with Type 2 Diabetes and Acute Coronary Syndrome. N Engl J Med 2015;373(23):2247-57.

5. Marso SP, Daniels GH, Brown-Frandsen K, et al. Liraglutide and Cardiovascular Outcomes in Type 2 Diabetes. N Engl J Med 2016;375(4):311-22.

6. Marso SP, Bain SC, Consoli A, et al. Semaglutide and Cardiovascular Outcomes in Patients with Type 2 Diabetes. N Engl J Med 2016;375(19):1834-44.

7. Holman RR, Bethel MA, George J, et al. Rationale and design of the EXenatide Study of Cardiovascular Event Lowering (EXSCEL) trial. Am Heart J 2016;174:103-10.

8. Mentz RJ, Hernandez AF, Berdan LG, et al. Good Clinical Practice Guidance and Pragmatic Clinical Trials: Balancing the Best of Both Worlds. Circulation 2016;133(9): 872-80.

9. Bentley-Lewis R, Aguilar D, Riddle MC, et al. Rationale, design, and baseline characteristics in Evaluation of LIXisenatide in Acute Coronary Syndrome, a long-term cardiovascular end point trial of lixisenatide versus placebo. Am Heart J 2015;169(5): 631-638.e7.
10. Marso SP, Poulter NR, Nissen SE, et al. Design of the liraglutide effect and action in diabetes: evaluation of cardiovascular outcome results (LEADER) trial. Am Heart J 2013;166(5):23-30.e5.

11. Samson SL, Garber AJ. A Plethora of GLP-1 Agonists: Decisions About What to Use and When. Curr Diab Rep 2016;16(12):120.

12. Mentz RJ, Cotter G, Cleland JG, et al. International differences in clinical characteristics, management, and outcomes in acute heart failure patients: better short-term outcomes in patients enrolled in Eastern Europe and Russia in the PROTECT trial. Eur J Heart Fail 2014;16(6):614-24.

13. Pfeffer MA, Claggett $B$, Assmann SF, et al. Regional variation in patients and outcomes in the Treatment of Preserved Cardiac Function Heart Failure With an Aldosterone Antagonist (TOPCAT) trial. Circulation 2015;131(1):34-42.

14. Scirica BM, Bhatt DL, Braunwald E, et al. Saxagliptin and cardiovascular outcomes in patients with type 2 diabetes mellitus. $\mathrm{N}$ Engl J Med 2013;369(14):1317-26.

15. Scirica BM, Braunwald E, Raz I, et al. Heart Failure, Saxagliptin, and Diabetes Mellitus: Observations from the SAVOR-TIMI 53 Randomized Trial. Circulation 2015;132(15):e198.

16. Zinman B, Wanner C, Lachin JM, et al. Empagliflozin, Cardiovascular Outcomes, and Mortality in Type 2 Diabetes. N Engl J Med 2015;373(22):2117-28.

17. Fitchett $D$, Zinman $B$, Wanner $C$, et al. Heart failure outcomes with empagliflozin in patients with type 2 diabetes at high cardiovascular risk: results of the EMPA-REG OUTCOME(R) trial. Eur Heart J 2016;37(19):1526-34. 